\title{
Outcomes of Total and Subtotal Laparoscopic Gastrectomy with D2 Lymphadenectomy in Advanced Gastric Cancer in a Brazilian Hospital
}

\author{
Augusto C. A. Tinoco1*, Matheus P. S. Netto1, Renam C. Tinoco1, Thammy L. Bastos', \\ Bárbara S. F. Paula1, Leonardo El-Kadre Tinoco², Luciana J. El-Kadre1 \\ ${ }^{1}$ Department of surgery, São José do Avaí Hospital, Itaperuna, Brazil \\ ${ }^{2}$ Iguaçu University, Itaperuna, Brazil \\ Email: ^augtinoco@gmail.com
}

How to cite this paper: Tinoco, A.C.A., Netto, M.P.S., Tinoco, R.C., Bastos, T.L., Paula, B.S.F., Tinoco, L.E. and El-Kadre, L.J. (2020) Outcomes of Total and Subtotal Laparoscopic Gastrectomy with D2 Lymphadenectomy in Advanced Gastric Cancer in a Brazilian Hospital. Surgical Science, 11, 166-176.

https://doi.org/10.4236/ss.2020.116019

Received: May 21, 2020

Accepted: June 21, 2020

Published: June 24, 2020

Copyright $\odot 2020$ by author(s) and Scientific Research Publishing Inc. This work is licensed under the Creative Commons Attribution International License (CC BY 4.0).

http://creativecommons.org/licenses/by/4.0/ (c) (i) Open Access

\begin{abstract}
Background: Although laparoscopic gastrectomy is becoming more popular as a curative therapy for gastric cancer, there are concerns about its oncological adequacy. We have compared the outcomes of laparoscopic total gastrectomy (LTG) and laparoscopic subtotal gastrectomy (LSG), both with modified D2 lymphadenectomies for the treatment of advanced gastric cancers. Aim: To compare the outcomes of laparoscopic (total and subtotal) gastrectomy with modified D2 lymphadenectomy for the treatment of gastric cancer, contributing to the literature regarding the overall survival of these patients and postoperative complications. Methods: From 1993 to 2014, 239 patients were operated on laparoscopic gastrectomy at our department. The routinely laparoscopic gastrectomy was performed in all patients with gastric cancer including those presenting with obstruction and bleeding. Data could be collected, on a retrospective way, from 2006 to 2014, from the medical records of 103 patients who underwent LSG $(n=72)$ or LTG $(n=31)$. We excluded patients with metastatic disease and those who could not have a complete tumor resection. Results: Most patients were in advanced stages of cancer. Adenocarcinoma was the most common find, with $43 \%$ of cases in stage IIA and $31 \%$ in stage IIIB. Intracorporeal Roux-en-Y or Billroth II anastomoses were employed. Postoperative complications, for LSG and LTG, were $18 \%$ and $35.4 \%$; mortality rate, during hospital stay, was $4.9 \%$ and $7.7 \%$; three-year survival rate, $53.1 \%$ and $59.3 \%$; and five-year survival rate, $46.9 \%$ and $40.7 \%$. Mean hospital stay was 7.08 days, being significantly lower in LSG group $(p<$ 0.05). Hospital acquired pneumonia was the most prevalent clinical complication, while deaths arising from surgical complications were caused mainly by gastro-jejunal or esophago-jejunal anastomosis leaks. Conclusions: Both
\end{abstract}


LSG and LTG with modified D2 lymphadenectomy are feasible alternatives to open surgery and survival rates were comparable. The increased risk of complications observed in LTG did not influence the overall mortality rate. We hope that these findings should contribute to improve the acceptance of laparoscopic gastrectomy as a safe procedure for gastric cancer treatment.

\section{Keywords}

Minimally Invasive Surgical Procedures, Gastric Neoplasms, Postoperative Period

\section{Introduction}

Gastric cancer remains one of the most common cancers worldwide, with high mortality. Although it is declining, its incidence, especially in Eastern Asia, is particularly high [1]. According to the American Cancer Society, it is estimated that 26,200 new cases of gastric cancer will be diagnosed in 2018 in the United States and approximately 10,800 people will die of this disease [2].

In Brazil, it is expected that 13,540 males and 7750 females will be affected by gastric cancer in 2018 [3]. Radical surgery is the only option for curative purposes in non-disseminated gastric cancer. Open surgery has long been the preferred surgical approach, associated with considerable morbidity and a postoperative mortality rate, ranging from $3.7 \%$ to $12.8 \%$ within 30 days postoperatively. This number is twice as high as the postoperative mortality rate found in patients with rectal cancer, which ranges from $0.9 \%$ to $7.5 \%$ [3] [4].

Interest in laparoscopic surgery of the gastrointestinal tract gathered momentum in the early 1990s, with the first report, describing laparoscopic colectomy, being published in 1991 [5]. In Latin America, the first laparoscopic subtotal gastrectomy, in a patient with gastric cancer, was reported in 1993, by Tinoco et al. [6]. Further pioneering studies were published soon afterwards describing, for example, laparoscopy-assisted Billroth I gastrectomy for the treatment of distal gastric cancer [7] and the first laparoscopy-assisted total gastrectomy [8]. The challenge of reproducing, with a different access, an established surgical technique deserves debate.

A number of more recent investigations have confirmed the viability and advantages of the laparoscopic approach for the management of early gastric cancer, with many reports showing that laparoscopic gastrectomy with D2 lymphadenectomy is feasible and produces minimal blood loss, despite the prolonged operative time [9] [10] [11] [12] [13].

On the other hand, on eastern settings, plenty of studies compared the efficacy of laparoscopic gastrectomy with open or video-assisted approaches. To the best of our knowledge, there are no reports comparing entirely laparoscopic total and subtotal gastrectomy (LTG and LSG, respectively) in the treatment of gastric cancer. Hence, we aimed to compare these two techniques with respect to loca- 
tion and type of tumor, postoperative complications, duration of hospitalization, mortality rate during hospitalization, and three- and five-year survival rates. The goal is to discuss both techniques, comparing the results, and not to address the indication of each type of reconstruction.

In addition to contributing to the overall understanding of the survival of these patients, the comparison of the two techniques may be a useful tool in the surgical decision in patients with bleeding or obstructive tumors.

\section{Materials and Methods}

The retrospective study was performed in accordance with the ethical standards of the Research Ethics Committee of Nova Iguaçu University, Rio de Janeiro, Brazil (registration number 24128619.1.0000.5288) and with 1964 Helsinki declaration and its later amendments. The C.M.O. of HSJA authorized access to the medical records of patients who had received either LSG or LTG for treatment of gastric cancer during the period 2006 to 2014.

Data collected included age of patient, histological classification of tumor, cancer staging, location of tumor, type of anastomoses, immediate postoperative complications, duration of hospitalization, mortality during hospitalization, and three- and five-year survival rates.

Since is a retrospective study, we were systematic in obtaining data for each patient. In those patients who were selected for the study and who had insufficient or discrepant data in the medical record, a telephone survey was applied for each case, resolving any doubts that existed.

Both male and female patients, with histological evidence of gastric cancer and fulfillment of resectability criteria were included in the study. The exclusion criteria used was: patients with metastatic disease and those who could not have a complete tumor resection (R1 resection).

There was no difference regarding demographic data in the distribution of the groups. Both had similar age distribution, similar comorbidities, and same ethnicity, most of them from the same macro-region (southeastern region of Brazil).

For the LTG group, patients with tumors in cardia, fundus and body were selected. In patients with body tumors, these should have a margin less than $<5 \mathrm{~cm}$ from the esophageal-gastric transition. For the LST group, patients with tumors in body and antrum were selected. These tumors should have a $5 \mathrm{~cm}$ margin above the macroscopic lesion and $2-3 \mathrm{~cm}$ margin below the pylorus.

In the LSG procedure, a side to side gastro-jejunal anastomosis was performed using a $45 \mathrm{~mm}$ endo linear stapler on the posterior gastric wall anastomosis. In the LTG procedure, esophago-jejunal anastomosis was done with a Medtronic $25 \mathrm{~mm}$ Endoscopic Intraluminal Stapler Tilt Top ${ }^{\mathrm{TM}}$ with the anvil connecting to nasogastric tube introduced through the mouth. Entero-entero Roux-en-Y anastomoses were carried out using a $45 \mathrm{~mm}$ White linear stapler.

The lymphadenectomy for gastric cancer were standardized with modified D2 lymphadenectomy, was performed the resection of stages D1+ 8a, 9, 10, 11p, 
11d, 12a for total gastrectomy, and D1+ 8a, 9, 10,11p, 12a for subtotal gastrectomy, both with splenic preservation (Figure 1, Figure 2).

Statistical analyses were performed using SPSS for Windows version 22.0 software with level of statistical significance set at 5\% $(p<0.05)$. The $\chi^{2}$ test was employed to compare the LTG and LSG groups of patients with respect to types of anastomosis, mortality rate during hospitalization, and three- and five-year postoperative survival rates. The Kolmogorov-Smirnov test was used to compare the two groups with regard to location and types of tumors, and the durations of hospitalization were compared using the Student $t$ test.

\section{Results}

From 1993 to 2014, 239 patients were operated on laparoscopic gastrectomy at our department. Data could be collected from 2006 to 2014, with 103 cases, 31 presenting with tumors of the cardia, fundus and body. Those patients were operated on LTG with distal esophagectomy and modified D2 lymphadenectomy $(\mathrm{D} 1+8 \mathrm{a}, 9,10,11 \mathrm{p}, 11 \mathrm{~d}, 12 \mathrm{a})$. In 72 cases tumors were in the body and antrum

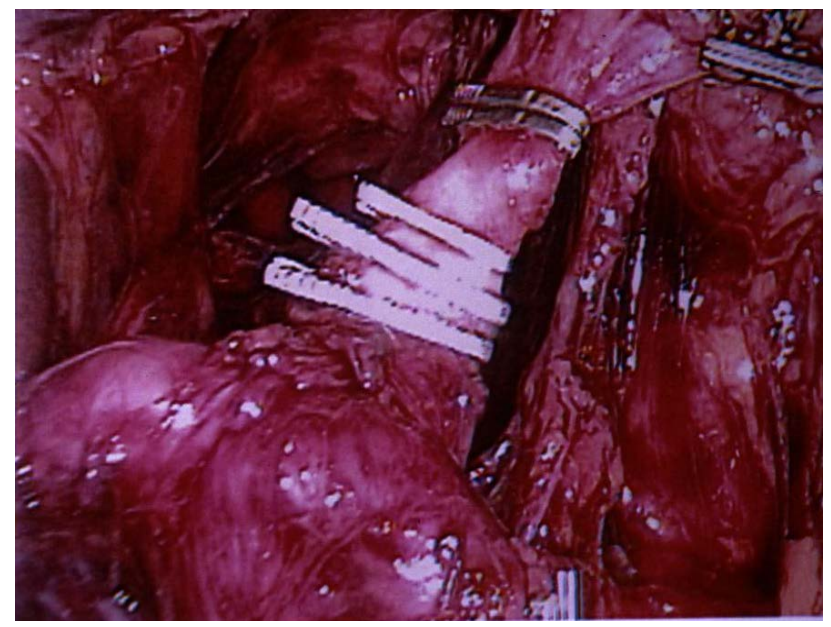

Figure 1. Left gastric artery lymphadenectomy.

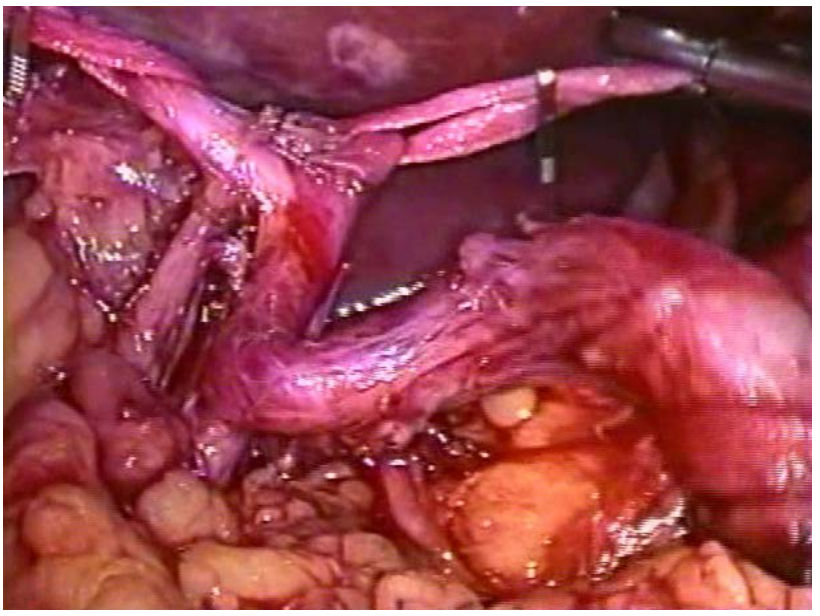

Figure 2. Hepatic artery lymphadenectomy. 
and LSG with modified D2 lymphadenectomy (D1+ 8a, 9, 10, 11p, 12a) was done.

Intracorporeal Roux-en-Y or Billroth II (BII) anastomoses were done to reconstruct the gastrointestinal tract.

Mean age was 65.6 years and $62.3 \%$ of the patients were male (Adenocarcinoma was the common find, mainly located in the antrum in the LSG group, and cardia and body, in the LTG group (Table 1).

Table 1. Comparison between patients submitted to LSG and LTG in a Brazilian setting.

\begin{tabular}{|c|c|c|c|}
\hline Variable & $\begin{array}{c}\text { LSG }(n=72) \\
n(\%)\end{array}$ & $\begin{array}{c}\text { LTG }(n=31) \\
n(\%)\end{array}$ & $p$ value \\
\hline Type of tumor & & & $>0.05$ \\
\hline Squamous cell carcinoma & - & $1(3.2)$ & \\
\hline MALT lymphoma & - & $1(3.2)$ & \\
\hline Carcinoma in situ & $1(1.4)$ & - & \\
\hline GIST & $11(15.3)$ & $1(3.2)$ & \\
\hline Adenocarcinoma & $60(83.3)$ & $28(90.3)$ & \\
\hline \multicolumn{4}{|l|}{ Location of tumor } \\
\hline Cardia & - & $11(35.5)$ & \\
\hline Body & $9(12.5)$ & $15(48.4)$ & \\
\hline Lesser curvature & $14(19.5)$ & $3(9.6)$ & \\
\hline Antrum & $49(68.0)$ & $2(6.5)$ & \\
\hline Types of anastomosis & & & $>0.05$ \\
\hline Roux-en-Y & $56(77.8)$ & $31(100)$ & \\
\hline Billroth II & $16(22.2)$ & 0 & \\
\hline Complications during hospitalization & & & $<0.05$ \\
\hline Pulmonary thromboembolism & $1(1.4)$ & - & \\
\hline Hematemesis/melena & $2(2.8)$ & - & \\
\hline Paralytic ileus & $1(1.4)$ & $1(3.2)$ & \\
\hline Cardiac complications & $1(1.4)$ & - & \\
\hline Duodenal stump fistula & $2(2.8)$ & - & \\
\hline Abdominal sepsis without fistula & $1(1.4)$ & - & \\
\hline Hospital acquired pneumonia & $3(4.2)$ & $6(19.3)$ & \\
\hline Gastro-jejunal anastomosis fistula & $2(2.8)$ & - & \\
\hline Esophago-jejunal anastomosis fistula & - & $4(12.9)$ & \\
\hline Postoperative survival & & & $>0.05$ \\
\hline Three-years & $38(53.1)$ & $18(59.3)$ & \\
\hline Five-years & $34(46.9)$ & $12(40.7)$ & \\
\hline
\end{tabular}

LSG = laparoscopic subtotal gastrectomy; LTG = laparoscopic total gastrectomy; MALT = mucosa-associated lymphoid tissue lymphoma; GIST = gastrointestinal stroma tumor. 
According to the criteria of the American Joint Committee on Cancer, the majority of patients were in advanced stage of cancer, with $43 \%$ in stage IIA, $17 \%$ in stage IIB, $9 \%$ in stage IIIA, and $31 \%$ in stage IIIB.

Roux-en-Y was preferred reconstruction in both groups. However, in the LST group, there were 16 patients (22.2\%) undergoing Billroth II reconstruction (Table 1).

A postoperative complication was found in $23.3 \%$ of the cases, in the LSG group, and $35.4 \%$ in the LTG one. For the LTG group, they were hospital acquired pneumonia in (19.3\%), esophago-jejunal anastomosis fistula in (12.9\%) and paralytic ileus in (3.2\%).

For the LSG group hospital acquired pneumonia occurred in (4.2\%), pulmonary thromboembolism in (1.4\%), hematemesis/melena in (2.8\%), paralytic ileus in (1.4\%), cardiac complication in (1.4\%), duodenal stump fistula IN (2.8\%), abdominal sepsis without fistula IN (1.4\%) and gastrojejunal anastomosis fistula IN (2.8\%).

The mean duration of hospitalization was 7.08 days, although the average stay of the LSG patients ( $6.02 \pm 1.82$ days) was significantly lower $(p<0.05)$ than that of LTG patients (9.18 \pm 3.53 days).

The mortality rate of LSG and LTG patients, during hospitalization, was $4.9 \%$ for LSG and $7.7 \%$ for LTG, with a mean rate of $5.8 \%$.

Deaths arising from surgical complications were caused by a duodenal stump leak in one patient and by an E-J leak in one case.

There were four leaks in the LSG, two in the duodenum stump and two on the gastrojejunal anastomosis. This complication occurred six, seven, nine and 11 days after surgery. From these cases, $75 \%$ of the patients presented with an albumin level $<3 \mathrm{mg} / \mathrm{dl}$.

For the LTG group, there were four leaks, all at the esophageal anastomosis, with two deaths. Leaks presented at days four, eight, nine and ten after surgery. From these patients, 50\% had an albumin level $<3 \mathrm{md} / \mathrm{dl}$.

The survival rates of LSG and LTG patients reached close to $60 \%$ at a three-year follow-up, and up to $47 \%$ at the five-year follow-up, with no significant differences between the two groups (Table 1).

\section{Discussion}

This study demonstrates the impact of two different surgical procedures in the surgical treatment of gastric cancer, focusing on early morbidity mortality and survival rate in 5 years. The importance of this article is related in the surgical indication for high risk patients, if is worth indicate the LGT or TLS with the same safety for the patients.

The LG complication rate was $23.3 \%$, close to the up limit found in the literature, ranging from $6 \%$ to $24 \%$. The hospital overall mortality rate was $5.8 \%$, which is within the range of $1 \%$ to $6 \%$ quoted in the literature, for a period of up to 30 days after surgery [14] [15]. Three explanations may be advanced to the 
relatively high levels of complication and mortality observed in our study population. 1) Patients treated at our hospital often presented with advanced stage tumors. All patients, over stage IIA; 2) Patients are undernourished and have no nutritional body reserves. There were four leaks in the LSG, two in the duodenum stump and two on the gastrojejunal anastomosis. From these cases, $75 \%$ of the patients presented with an albumin level $<3 \mathrm{mg} / \mathrm{dl}$; 3) patients over 70 years had a significantly higher risk of morbidity and mortality. In our data, 45 patients (43.6\%) were older than 70 years.

Papenfuss et al., comparing open total and subtotal gastrectomies in 2.580 patients, found higher morbidity and 30-day-mortality rates after total gastrectomy $(29.3 \%$ vs. $19.9 \%, p<0.001$; and 5.4 vs. $3.4 \%, p<0.015)[16]$.

Our study showed a higher morbidity with LTG. On the other hand, the 30 -day-mortality ( $7.7 \%$ and $4.9 \%$ respectively) was not significantly different. The laparoscopic approach may contribute to a better outcome, regarding 30-day-mortality, when compared to open surgery.

Another paper, investigating age-related differences in surgically treated patients with gastric cancer, showed, in 2315 cases, a high 30-day mortality rate (7.9\%), occurring in patients over 70 years [17].

For eastern surgeons, the conventional operation is considered to be outdated. Therefore, in Japan, China and South Korea, where incidence of gastric cancer occurrence is higher than the rest of the world, laparoscopic gastrectomy is considered to be a safe and effective surgery [18] [19]. However, the controversy about the suitability of laparoscopy as a definitive treatment remains, especially for cases of advanced cancer where OG plays an important role.

Nevertheless, LG has become popular based on several prospective randomized controlled trials (RCTs) that reported improved short-term surgical outcomes with similar oncological safety to that of OG [20] [21] [22].

OG has improved considerably over the years and replication of achievable outcomes is a challenge for the supporters of minimally invasive laparoscopy. Indeed, there are many barriers to be overcome, including a steep learning curve.

The technical difficulties associated with LSG and LTG are related mainly to lymphadenectomy and the risk of damaging blood vessels and other structures during lymph node dissection. Moreover, reconstruction of the intestinal tract demands extensive experience of advanced laparoscopic techniques, suturing, handling of staplers and manipulation of energy-driven tweezers. The facility with which such skills may be acquired by the surgeon depends, in part, on the size of the hospital/service and the flow of patients.

Thus, institutions that care for a larger number of gastric cancer cases tend to exhibit better results than those with a more limited flow [23] [24] [25] [26].

The three- and five-year survival rates of patients treated at our hospital (Table 1) are within the expected values for gastric cancer surgeries, although data in the literature are variable for such cases. 
According to the American Cancer Society, the global five-year survival rate ranges from $36 \%$, for patients at the IIIB stage, to $82 \%$, for patients at the IIA stage [27].

We believe that the rates reported herein are comparable with those achieved by conventional surgery, especially considering that $31 \%$ of the patients were on stage IIIB, and $43.6 \%$ were older than $70 \mathrm{y}$.

Malnourished patients are at greater risk of adverse clinical outcomes and proper nutrition before surgery may improve recovery after gastrectomy. In the majority of patients, efforts to improve nutritional status pre-operatively, or to apply nutritional interventions, could not be done, considering the advanced stage of the disease, either with obstruction or with bleeding.

None of these patients were submitted to neoadjuvant chemotherapy, which could positively impact survival. Late gastric cancer diagnosis in the western setting, with an increased incidence of advanced stages, may propose the discussion about delaying the only chance of curative management.

Moreover, laparoscopic approach, on eastern series, was mostly used for less advanced cases, below $\mathrm{T} 2$ stage.

The success of our surgical team in treating gastric cancer patients may be attributed to the experience acquired in advanced laparoscopy gained mainly through the performance of bariatric surgery, an intervention that has been practiced in our service for the last two decades. Furthermore, it is acknowledged that laparoscopic gastrectomy, together with D2 lymphadenectomy, increases the chances of survival [28] [29]. For this reason, the laparoscopic procedure is the standard one in our hospital, with no increase in the mortality rate, compared with that of OG.

In addition, the evolution of instrumentation employed in laparoscopic surgery has led to more precise lymph node dissection. This allowed a clearer identification of lymph node stations along the left gastric, antero-superior hepatic and splenic arteries (stations 7, 8a and 11) and directly into the celiac trunk (station 9).

In our hospital, the lymphadenectomy for gastric cancer were standardized with modified D2 lymphadenectomy, where we performed the resection of stages $\mathrm{D} 1+8 \mathrm{a}, 9,10,11 \mathrm{p}, 11 \mathrm{~d}, 12 \mathrm{a}$ for total gastrectomy, and D1+ 8a, 9, 10,11p, 12a for subtotal gastrectomy, both with splenic preservation [30].

The average hospital stay was 7.08 days, being comparable to the reported one in the literature $(8.11 \pm 4.10$ days $)$ [31]. For LTG patients, group with more postoperative complications, it was 9.18 days.

\section{Conclusions}

Our experience demonstrated that LSG and LTG are feasible alternatives to OG, and both procedures afforded comparable survival rates. Although patients submitted to LTG presented a higher level of early postoperative complications, it did not influence the long-term survival rate. 
Overall, the results of morbidity and mortality were similar to those of OG, with the advantages of a minimally invasive technique. Our study may represent a contribution in the field of laparoscopic surgery, considering that the exchange of information between multiple hospital centers worldwide can favor the laparoscopy approach for gastric cancer.

Although OG has a long history of improvement and achievement, remaining the gold standard procedure in many cases, there should be emphasized that surgical procedures need to be constantly challenged, and may need to be modified or replaced, according to the individual needs of the patient.

\section{Conflicts of Interest}

The authors declare that they have no conflict of interests.

\section{References}

[1] Bertuccio, P., Chatenoud, L., Levi, F., et al. (2009) Recent Patterns in Gastric Cancer: A Global Overview. International Journal of Cancer, 125, 666-673. https://doi.org/10.1002/ijc.24290

[2] Siegel, R.L., Miller, K.D. and Jemal, A. (2019) Cancer Statistics, 2019. CA: A Cancer Journal for Clinicians, 69, 7-34. https://doi.org/10.3322/caac.21551

[3] Van Gestel, Y.R.B.M., Lemmens, V.E.P.P., De Hingh, I.H.J.T., et al. (2013) Influence of Comorbidity and Age on 1-, 2-, and 3-Month Postoperative Mortality Rates in Gastrointestinal Cancer Patients. Annals of Surgical Oncology, 20, 371-380. https://doi.org/10.1245/s10434-012-2744-1

[4] Busweiler, L.A.D., Wijnhoven, B.P.L., van Berge Henegouwen, M.I., et al. (2016) Early Outcomes from the Dutch Upper Gastrointestinal Cancer Audit. British Journal of Surgery, 103, 1855-1863. https://doi.org/10.1002/bjs.10303

[5] Jacobs, M., Verdeja, J.C. and Goldstein, H.S. (1991) Minimally Invasive Colon Resection (Laparoscopic Colectomy). Surgical Laparoscopy \& Endoscopy, 1, 144-150.

[6] Tinoco, R., Tinoco, A., Linhares, E., Lourenço, L. and Sano, T. (2005) A laparoscopia no câncer gástrico. In: Editora, T., Ed., Atualização em Câncer Gástrico ( $A$ laparoscopia no câncer gástrico), São Paulo, 243-249.

[7] Kitano, S., Iso, Y., Moriyama, M. and Sugimachi, K. (1994) Laparoscopy-Assisted Billroth I Gastrectomy. Surgical Laparoscopy \& Endoscopy, 4, 146-148.

[8] Azagra, J.S., Goergen, M., De Simone, P. and Ibanez-Aguirre, J. (1999) Minimally Invasive Surgery for Gastric Cancer. Surgical Endoscopy, 13, 351-357. https://doi.org/10.1007/s004649900988

[9] Uyama, I., Sugioka, A., Matsui, H., Fujita, J., Komori, Y. and Hasumi, A. (2000) Laparoscopic D2 Lymph Node Dissection for Advanced Gastric Cancer Located in the Middle or Lower Third Portion of the Stomach. Gastric Cancer, 3, 50-55. https://doi.org/10.1007/PL00011690

[10] Tanimura, S., Higashino, M., Fukunaga, Y., et al. (2008) Laparoscopic Gastrectomy for Gastric Cancer: Experience with More than 600 Cases. Surgical Endoscopy, 22, 1161-1164. https://doi.org/10.1007/s00464-008-9786-2

[11] Kawamura, H., Homma, S., Yokota, R., et al. (2008) Inspection of Safety and Accuracy of D2 Lymph Node Dissection in Laparoscopy-Assisted Distal Gastrectomy. World Journal of Surgery, 32, 2366-2370.

https://doi.org/10.1007/s00268-008-9697-3 
[12] Song, K.Y., Kim, S.N. and Park, C.H. (2008) Laparoscopy-Assisted Distal Gastrectomy with D2 Lymph Node Dissection for Gastric Cancer: Technical and Oncologic Aspects. Surgical Endoscopy, 22, 655-659. https://doi.org/10.1007/s00464-007-9431-5

[13] Lee, J.H., Kim, Y.W., Ryu, K.W., et al. (2007) A Phase-II Clinical Trial of Laparoscopy-Assisted Distal Gastrectomy with D2 Lymph Node Dissection for Gastric Cancer Patients. Annals of Surgical Oncology, 14, 3148-3153. https://doi.org/10.1245/s10434-007-9446-0

[14] Andreollo, N.A., Lopes, L.R. and Coelho Neto, J.S. (2011) Postoperative Complications after Total Gastrectomy in the Gastric Cancer: Analysis of 300 Patients. Arquivos Brasileiros de Cirurgia Digestiva (São Paulo), 24, 126-130.

[15] Huang, Y.-L., Lin, H.G., Yang, J.-W., et al. (2014) Laparoscopy-Assisted versus Open Gastrectomy with D2 Lymph Node Dissection for Advanced Gastric Cancer: A Meta-Analysis. International Journal of Clinical and Experimental Medicine, 7, 1490-1499.

[16] Papenfuss, W.A., Kukar, M., Oxenberg, J., et al. (2014) Morbidity and Mortality Associated with Gastrectomy for Gastric Cancer. Annals of Surgical Oncology, 21, 3008-3014. https://doi.org/10.1245/s10434-014-3664-Z

[17] Nelen, S.D., Bosscha, K., Lemmens, V.E.P.P., Hartgrink, H.H., Verhoeven, R.H.A. and de Wilt, J.H.W. (2018) Morbidity and Mortality According to Age Following Gastrectomy for Gastric Cancer. British Journal of Surgery, 105, 1163-1170. https://doi.org/10.1002/bjs.10836

[18] Yasunaga, H., Horiguchi, H., Kuwabara, K., et al. (2013) Outcomes after Laparoscopic or Open Distal Gastrectomy for Early-Stage Gastric Cancer: A Propensity-Matched Analysis. Annals of Surgery, 257, 640-646. https://doi.org/10.1097/SLA.0b013e31826fd541

[19] Lin, J.X., Huang, C.M., Zheng, C.H., et al. (2015) Surgical Outcomes of 2041 Consecutive Laparoscopic Gastrectomy Procedures for Gastric Cancer: A Large-Scale Case Control Study. PLoS ONE, 10, e0114948. https://doi.org/10.1371/journal.pone.0114948

[20] Hayashi, H., Ochiai, T., Shimada, H. and Gunji, Y. (2005) Prospective Randomized Study of Open versus Laparoscopy-Assisted Distal Gastrectomy with Extraperigastric Lymph Node Dissection for Early Gastric Cancer. Surgical Endoscopy, 19, 1172-1176. https://doi.org/10.1007/s00464-004-8207-4

[21] Huscher, C.G.S., Mingoli, A., Sgarzini, G., et al. (2005) Laparoscopic versus Open Subtotal Gastrectomy for Distal Gastric Cancer: Five-Year Results of a Randomized Prospective Trial. Annals of Surgery, 241, 232-237. https://doi.org/10.1097/01.sla.0000151892.35922.f2

[22] Kitano, S., Shiraishi, N., Fujii, K., Yasuda, K., Inomata, M. and Adachi, Y. (2002) A Randomized Controlled Trial Comparing Open vs Laparoscopy-Assisted Distal Gastrectomy for the Treatment of Early Gastric Cancer: An Interim Report. Surgery, 131, S306-S311. https://doi.org/10.1067/msy.2002.120115

[23] Zeng, Y.K., Yang, Z.L., Peng, J.S., Lin, H.S. and Cai, L. (2012) Laparoscopy-Assisted versus Open Distal Gastrectomy for Early Gastric Cancer: Evidence from Randomized and Nonrandomized Clinical Trials. Annals of Surgery, 256, 39-52. https://doi.org/10.1097/SLA.0b013e3182583e2e

[24] Shimizu, S., Uchiyama, A., Mizumoto, K., et al. (2000) Laparoscopically Assisted Distal Gastrectomy for Early Gastric Cancer: Is It Superior to Open Surgery? Surgical Endoscopy, 14, 27-31. https://doi.org/10.1007/s004649900005 
[25] Songun, I., Putter, H., Kranenbarg, E.M.K., Sasako, M. and van de Velde, C.J.H. (2010) Surgical Treatment of Gastric Cancer: 15-Year Follow-Up Results of the Randomised Nationwide Dutch D1D2 Trial. The Lancet Oncology, 11, 439-449. https://doi.org/10.1016/S1470-2045(10)70070-X

[26] Anderson, O., Ni, Z., Møller, H., et al. (2011) Hospital Volume and Survival in Oesophagectomy and Gastrectomy for Cancer. European Journal of Cancer, 47, 2408-2414. https://doi.org/10.1016/j.ejca.2011.07.001

[27] Stomach Cancer Survival Rates. The American Cancer Society Medical and Editorial Content Team.

https://www.cancer.org/cancer/stomach-cancer/detection-diagnosis-staging/surviva l-rates.html

[28] Jiang, L., Yang, K.H., Chen, Y., et al. (2014) Systematic Review and Meta-Analysis of the Effectiveness and Safety of Extended Lymphadenectomy in Patients with Resectable Gastric Cancer. British Journal of Surgery, 101, 595-604.

https://doi.org/10.1002/bjs.9497

[29] De Steur, W.O., Dikken, J.L. and Hartgrink, H.H. (2013) Lymph Node Dissection in Resectable Advanced Gastric Cancer. Digestive Surgery, 30, 96-103. https://doi.org/10.1159/000350873

[30] Eshuis, W.J., Van Berge, M.I., Werner, H. and Gisbertz, S.S. (2018) Compliance to D2 Lymphadenectomy in Laparoscopic Gastrectomy. Updates in Surgery, 70, 197-205. https://doi.org/10.1007/s13304-018-0553-1

[31] Kang, B.H., Xuan, Y., Hur, H., Ahn, C.W., Cho, Y.K. and Han, S.U. (2012) Comparison of Surgical Outcomes between Robotic and Laparoscopic Gastrectomy for Gastric Cancer: The Learning Curve of Robotic Surgery. Journal of Gastric Cancer, 12, 156-163. https://doi.org/10.5230/jgc.2012.12.3.156 